\title{
Balance assessment in healthy children and adolescents aged $6-18$ years based on six tests collected on AMTI AccuSway force platform
}

\author{
KRZYSZTOF GRAFF ${ }^{1,2}$, EWA SZCZERBIK ${ }^{1}$, MAŁGORZATA KALINOWSKA ${ }^{1}$, \\ MACIEJ JAWORSKI ${ }^{3}$, MAŁGORZATA SYCZEWSKA ${ }^{1 *}$ \\ ${ }^{1}$ Department of Rehabilitation, The Children's Memorial Health Institute, Warsaw, Poland. \\ ${ }^{2}$ Department of Rehabilitation, Józef Piłsudski Univeristy of Physical Education in Warsaw, Warsaw, Poland. \\ ${ }^{3}$ Department of Biochemistry, Radioimmunology and Experimental Medicine, \\ The Children's Memorial Health Institute, Warsaw, Poland.
}

\begin{abstract}
Purpose: The aim of the study was to compare the results of six balance tests collected on AMTI AccuSway Plus ACS force platform between healthy female and male children and adolescents. We also searched for possible correlation of the balance measures with subjects' age. Methods: 228 healthy 6- to 18-year-old subjects (111 boys and 117 girls) participated in the study. Six balance tests were performed with the use of AMTI AccuSway Plus ACS platform: quiet standing for 30s, maximal voluntary sways of the body in the sagittal plane (anterior-posterior - AP test) for $30 \mathrm{~s}$, and in the frontal plane (left-right - ML test) for 30s. All tests were performed in two conditions: eyes open and eyes closed. Results: During quiet standing with eyes open, most balance measures were lower in girls $(p<0.05)$. In AP and ML tests with eyes open, a few balance parameters were different between boys and girls $(p<0.05)$. In quiet standing, AP and ML tests with eyes closed, there were no between-gender differences $(p>0.05)$. In quiet standing with eyes open and closed most balance parameters were negatively correlated with age $(p<0.05)$. Conclusions: Quiet standing postural sway characteristics depended on gender under normal visual conditions and it was similar in boys and girls under visual deprivation conditions. The vision was differently used by females and males in balance tasks. Static postural stability improved with age regardless of visual conditions.
\end{abstract}

Key words: balance, reference data of healthy children, children and adolescents

\section{Introduction}

Proper body posture and balance are required for all daily activities, sport trainings and for avoiding of falls. Development of balance starts early in life and any disturbance of it can have serious impact not only for keeping upright posture properly, but also on other motor functions, such as gait. The balance control develops during childhood and adolescence gradually. During first period, from birth to the acquisition of upright stance, control appears first at the neck muscles, later trunk muscles, and at the end in the legs muscles. During the second period, from upright stance till approximately 6 years of age a coordination between lower and upper parts of the body develops. The third phase starts around 7th year of age, and continuous till the adulthood [1]. There are

\footnotetext{
* Corresponding author: Małgorzata Syczewska, Department of Rehabilitation, The Children's Memorial Health Institute, al. Dzieci Polskich 20,04-730 Warsaw, Poland, Phone: +48-22-8151748, e-mail: m.syczewska@ipczd.pl

Received: November 4th, 2019

Accepted for publication: April 16th, 2020
} 
hypotheses pointing out that babies and children use different combinations of sensory information to maintain proper balance. Three classes of sensory information are used for balance control: visual, vestibular, and somatosensory. During babyhood and childhood, vison predominates in balance control, and around 7th year of age vestibular contribution starts to dominate [1].

During childhood and adolescence balance disturbances can be first signs of serious diseases, for example one of the early signs of the brain tumours are frequent falls. Therefore, balance assessment is often used in many clinical situations: after injuries, when suspicion of neurodegenerative disorders arise, when proprioceptive problems are present [5], [12], [16], or to assess the progress of the rehabilitation treatment [2]. The balance abilities of the children and adolescents can be also assessed in subjects training various sport activities [11].

To properly evaluate the results of the balance test of a patient, a normative data are required. The results of the patient are being compared to this database in order to conclude presence or absence of balance problems. During the maturation process in children (and to some extent in adolescents), the central nervous system matures, the body proportions and mass distribution change, and the centre of mass moves upwards [6]. Some studies show that regarding the balance control there is no difference between the sexes, but others describe the developmental differences between boys and girls [7], [12], [15]. Therefore, the normative database should consider possible differences between sexes, and influence of age [8]-[10], [12], taking also the developmental process of balance control into account [1].

There are many commercially available posturographic systems on the market, with many different static and dynamic tests [3], [13]. One of the widely used balance platforms is AMTI AccuSway Plus ACS. This force platform measures position of centre of pressure $(\mathrm{CoP})$ during standing and calculates several parameters from the CoP fluctuations in time. The platform enables performance of several balance tests during the patient's assessment.

The aim of the study was to compare the results of six balance tests collected on AMTI AccuSway Plus ACS force platform between healthy female and male children and adolescents. We also searched for possible correlation of the balance measures with subjects' age. The results of this study may serve as a reference data base for clinical purposes.

\section{Materials and methods}

\subsection{Participants}

228 healthy children and adolescents (111 boys and 117 girls) aged 6 to 18 participated in the study. The questionnaire and clinical evaluation made by experienced physiotherapist ensured that only healthy subjects participated in the study. All orthopaedic or neurological problems, as well as previous injuries were exclusion criteria. Body mass and body height were measured, and BMI calculated. Only one subject was slightly overweighed. The demographic data of the participants are given in Table 4. The study was approved by Local Ethical Committee. The subjects were recruited in Warsaw schools, in agreement with schools' authorities. The parents and guardians were informed about the purpose of the study and gave their informed consent.

\subsection{Design and procedures}

The balance was evaluated using AMTI AccuSway Plus ACS platform with Balance Clinic 1.4.2 software (sampling frequency was $50 \mathrm{~Hz}$, no filter was used). All subjects underwent six balance tests: quiet standing for $30 \mathrm{~s}$, maximal voluntary sways of the body in sagittal plane - antero-posterior (AP test) for $30 \mathrm{~s}$, and in frontal plane - left-right (mediolateral, ML test) for $30 \mathrm{~s}$. All these tests were performed in two conditions: eyes open and eyes closed. The subjects were standing with their feet parallel to each other (barefoot), with distance between them approximately equal to the pelvic width, upper extremities freely hanging along the trunk. During the tests with eyes open they were fixing their gaze in front of them, on the wall. The subjects were instructed to sway using primarily their ankles (voluntary sways in sagittal plane) without rising heels or toes from the ground, and to shift their body weight from left to right limb without detaching feet from the ground (voluntary sways in frontal plane). They chose the frequency of the sways themselves. They were also instructed to sway as far as they could, feeling safely. Short breaks were allowed between the tests. The list of assessed variables is given:

- $X$ Max - maximal displacement of COP to the right $[\mathrm{cm}]$,

- $X$ Min - maximal displacement of COP to the left $[\mathrm{cm}]$, 
- Symmetry $X-X \operatorname{Max} / X \operatorname{Min}$,

- $Y$ Max - maximal forward displacement of COP $[\mathrm{cm}]$,

- $Y$ Min - maximal backward displacement of COP [cm],

- Symmetry $Y-Y \operatorname{Max} / Y$ Min,

- Range $X$ - range of COP displacement in frontal plane $[\mathrm{cm}]$,

- Range $Y$ - range of COP displacement in sagittal plane $[\mathrm{cm}]$,

- Circular sway area - area of the circular sway area $\left[\mathrm{cm}^{2}\right]$,

- Path length - length of the path covered by COP during the test $[\mathrm{cm}]$,

- $V$ Avg - mean velocity of COP during the test $[\mathrm{cm} / \mathrm{s}]$,

- Area 95 - area covering $95 \%$ of the COP sway,

- WCOP - COP oscillation index, calculated according to the formula [10]:

$$
\begin{aligned}
& \text { WCOP }=100 X[(\text { Range } X-\text { Range } Y) / \\
& \quad(\text { Range } X+\text { Range } Y)]
\end{aligned}
$$

- $\mathrm{WCOP}=0$ - no dominance in any plane,

- WCOP $>0$ - dominance of COP sway in sagittal plane,

- WCOP $<0$ - dominance of COP sway in frontal plane.

\subsection{Statistical analysis}

The data collected from all tests were put into the spreadsheet, and statistical calculations were performed using STATISTICA v.10.0 (StatSoft) software. To check the normality of the variables the chi-square test was used. As most of the variables were nonnormally distributed the differences between the sexes were checked with Mann-Whitney $U$ nonparametric test (whole base and in age groups), and the dependence on age was evaluated with Spearman's Rank Correlation Coefficient. The cut-off value of $p$-level was 0.05 . The strength of the statistically significant correlations was assessed using conventional approach [14].

\section{Results}

With eyes open, the following parameters depended on gender during quiet standing: medio-lateral and fore-aft sway $(X \operatorname{Max} p=0.006, X \operatorname{Min} p=0.004$, $Y \operatorname{Max} p=0.001, Y \operatorname{Min} p=0.007, \operatorname{Sum} X p=0.003$, Sum $Y p=0.003$ ), together with sway area (Circular sway area $p<0.001$, Area95 $p<0.001$ ).

During AP test with eyes open only three variables depended on gender: medio-lateral sway $(X$ Max $p=$ $0.042)$, COP velocity (WCOP_V $p=0.034$ ) and path length (Path Length $p=0.033$ ). In the case of ML test, only path length depended on gender (Path Length $p=0.035$ ).

In all three tests (quiet standing, ML test, and AP test), there was no statistically significant difference between genders in eyes closed condition, apart from mean velocity in ML test $(p=0.047)$.

In quiet standing with eyes open and eyes closed, most parameters of boys and girls weakly or moderately negatively correlated with age. For the entire group of the study participants, path length and mean velocity strongly negatively correlated with age. In ML and AP tests, most parameters weakly and positively correlated with age. The correlations between balance test variables and age (with gender factor considered) are presented in Table 1 (quiet standing), Table 2 (ML test) and Table 3 (AP test).

In Table 4, the demographic data in sex and age subgroups are presented. The data were summarized in Tables 5-7 as medians and 5\% and 95\% percentiles of the balance parameters. Depending on the dependence on age and/or gender, the data were divided into age/gender subgroups.

Table 1. Significant correlation coefficients $(r)$ of balance measures with age.

\begin{tabular}{|c|c|c|c|c|}
\hline \multirow[t]{2}{*}{ Variable } & $\begin{array}{l}r-\text { boys } \\
N=111\end{array}$ & $\begin{array}{l}r-\text { girls } \\
N=117\end{array}$ & $\begin{array}{c}r \text { together } \\
N=228\end{array}$ & \multirow{2}{*}{\begin{tabular}{|c}
$\begin{array}{c}r \text { together } \\
N=228\end{array}$ \\
Eyes closed
\end{tabular}} \\
\hline & \multicolumn{3}{|c|}{ Eyes open } & \\
\hline$X \operatorname{Max}$ & -0.375 & -0.373 & - & -0.370 \\
\hline$X$ Min & 0.285 & 0.400 & - & 0.337 \\
\hline Symmetry $X$ & - & - & ns & ns \\
\hline$Y \operatorname{Max}$ & -0.386 & -0.39 & - & -0.314 \\
\hline$Y$ Min & 0.351 & 0.473 & - & 0.284 \\
\hline Symmetry $Y$ & - & - & ns & ns \\
\hline Range $X$ & -0.359 & -0.395 & - & -0.390 \\
\hline Range $Y$ & -0.394 & -0.423 & - & -0.319 \\
\hline Circular sway area & -0.435 & -0.470 & - & -0.419 \\
\hline PathLength & - & - & -0.875 & -0.813 \\
\hline$V$ Avg & - & - & -0.883 & -0.798 \\
\hline Area95 & -0.339 & -0.427 & - & -0.371 \\
\hline WCOP & - & - & ns & 0.156 \\
\hline
\end{tabular}

Test: quiet standing for $30 \mathrm{~s}$, eyes open and eyes closed

ns - non significant. 
Table 2. Significant correlation coefficients $(r)$ of balance measures with age.

Test: AP test, eyes open and eyes closed

\begin{tabular}{|c|c|c|c|c|}
\hline \multirow[t]{2}{*}{ Variable } & $\begin{array}{l}r-\text { boys } \\
N=111\end{array}$ & $\begin{array}{l}r-\text { girls } \\
N=117\end{array}$ & $\begin{array}{c}r \text { together } \\
N=228\end{array}$ & \multirow{2}{*}{\begin{tabular}{|c}
$r$ together \\
$N=228$ \\
Eyes closed
\end{tabular}} \\
\hline & \multicolumn{3}{|c|}{ Eyes open } & \\
\hline$X$ Max & -0.247 & -0.460 & - & -0.329 \\
\hline$X$ Min & - & - & 0.342 & 0.342 \\
\hline Symmetry $X$ & - & - & ns & ns \\
\hline$Y$ Max & - & - & 0.438 & 0.347 \\
\hline$Y$ Min & - & - & -0.552 & -0.442 \\
\hline Symmetry $Y$ & - & - & 0.292 & ns \\
\hline Range $X$ & - & - & -0.385 & -0.338 \\
\hline Range $Y$ & - & - & 0.527 & 0.431 \\
\hline Circular sway area & - & - & -0.140 & ns \\
\hline PathLength & ns & ns & - & ns \\
\hline V Avg & - & - & ns & ns \\
\hline Area95 & - & - & ns & $\mathrm{ns}$ \\
\hline WCOP & - & - & 0.613 & 0.503 \\
\hline
\end{tabular}

ns - non significant.
Table 3. Significant correlation coefficients $(r)$ of balance measures with age.

Test: AP test, eyes open and eyes closed

\begin{tabular}{|l|c|c|}
\hline \multirow{2}{*}{ Variable } & $\begin{array}{c}r \text { together } \\
N=228\end{array}$ & $\begin{array}{c}r \text { together } \\
N=228\end{array}$ \\
\cline { 2 - 3 } & Eyes open & Eyes closed \\
\hline$X$ Max & 0.367 & 0.308 \\
\hline$X$ Min & -0.347 & -0.313 \\
\hline Symmetry $X$ & $\mathrm{~ns}$ & $\mathrm{~ns}$ \\
\hline$Y$ Max & -0.145 & $\mathrm{~ns}$ \\
\hline$Y$ Min & $\mathrm{ns}$ & $\mathrm{ns}$ \\
\hline Symmetry $Y$ & 0.137 & 0.187 \\
\hline Range $X$ & 0.378 & 0.306 \\
\hline Range $Y$ & $\mathrm{~ns}$ & $\mathrm{~ns}$ \\
\hline Circular sway area & 0.175 & $\mathrm{~ns}$ \\
\hline PathLength & $\mathrm{ns}$ & $\mathrm{ns}$ \\
\hline$V$ Avg & $\mathrm{ns}$ & $\mathrm{ns}$ \\
\hline Area95 & 0.252 & 0.187 \\
\hline WCOP & 0.353 & $\mathrm{~ns}$ \\
\hline
\end{tabular}

ns - non significant.

Table 4. Demographic data of 228 healthy children and adolescents divided into age and sex subgroups. Data are presented as means \pm standard deviations

\begin{tabular}{|c|c|c|l|c|c|}
\hline \multicolumn{1}{|c|}{ Age groups } & Boys $(n)$ & Girls $(n)$ & \multicolumn{1}{c|}{ Variable } & Boys & Girls \\
\hline 6-9 [years] & 37 & 32 & Body mass [kg] & $27.1 \pm 6.9$ & $29.3 \pm 7.7$ \\
\hline & & & Body height [m] & $1.28 \pm 0.10$ & $1.31 \pm 0.11$ \\
\hline & & & BMI & $16.4 \pm 2.5$ & $17.2 \pm 2.7$ \\
\hline $10-13$ [years] & 33 & 36 & Body mass [kg] & $43.2 \pm 10.1$ & $44.8 \pm 10.1$ \\
\hline & & & Body height [m] & $1.51 \pm 0.07$ & $1.52 \pm 0.07$ \\
\hline & & & BMI & $18.9 \pm 2.2$ & $19.1 \pm 3.3$ \\
\hline $14-18$ [years] & 41 & 49 & Body mass [kg] & $69.4 \pm 13.5$ & $58.1 \pm 10.2$ \\
\hline & & & Body height [m] & $1.77 \pm 0.08$ & $1.66 \pm 0.07$ \\
\hline & & & BMI & $22.2 \pm 3.4$ & $21.1 \pm 3.2$ \\
\hline
\end{tabular}

Table 5. Median and 5\% and $95 \%$ percentiles of the balance variables

in group of 228 healthy subjects ( 111 boys and 117 girls). Test: quiet standing for $30 \mathrm{~s}$

\begin{tabular}{|c|c|c|c|c|c|}
\hline \multirow{2}{*}{ Variable } & Age [years] & Boys & Girls & Together & Together \\
\hline & \multicolumn{4}{|c|}{ Eyes open } & Eyes closed \\
\hline 1 & 2 & 3 & 4 & 5 & 6 \\
\hline \multirow[t]{3}{*}{$X \operatorname{Max}[\mathrm{cm}]$} & $6-9$ & $\begin{array}{l}1.090 \\
0.530 ; 1.910 \\
\end{array}$ & $\begin{array}{l}0.970 \\
0.410 ; 1.490\end{array}$ & - & $\begin{array}{l}1.250 \\
0.640 ; 1.940\end{array}$ \\
\hline & $10-13$ & $\begin{array}{l}0.730 \\
0.350 ; 1.580 \\
\end{array}$ & $\begin{array}{l}0.740 \\
0.390 ; 1.380 \\
\end{array}$ & - & $\begin{array}{l}0.810 \\
0.460 ; 1.930 \\
\end{array}$ \\
\hline & $14-18$ & $\begin{array}{l}0.740 \\
0.280 ; 1.030\end{array}$ & $\begin{array}{l}0.475 \\
0.280 ; 1.220\end{array}$ & - & $\begin{array}{l}0.720 \\
0.360 ; 1.550\end{array}$ \\
\hline \multirow[t]{3}{*}{$X \operatorname{Min}[\mathrm{cm}]$} & $6-9$ & $\begin{array}{l}-0.810 \\
-3.200 ;-0.490\end{array}$ & $\begin{array}{l}-0.845 \\
-1.980 ;-0.450\end{array}$ & - & $\begin{array}{l}-1.070 \\
-2.560 ;-0.680\end{array}$ \\
\hline & $10-13$ & $\begin{array}{l}-0.720 \\
-1.500 ;-0.390\end{array}$ & $\begin{array}{l}-0.635 \\
-1.060 ;-0.390\end{array}$ & - & $\begin{array}{l}-0.820 \\
-1.650 ;-0.350\end{array}$ \\
\hline & $14-18$ & $\begin{array}{l}-0.690 \\
-1.210 ;-0.310\end{array}$ & $\begin{array}{l}-0.550 \\
-1.050 ;-0.240\end{array}$ & - & $\begin{array}{l}-0.720 \\
-1.430 ;-0.360\end{array}$ \\
\hline
\end{tabular}




\begin{tabular}{|c|c|c|c|c|c|}
\hline 1 & 2 & 3 & 4 & 5 & 6 \\
\hline Symmetry $X$ & $6-18$ & - & - & \begin{tabular}{|l|}
-104.545 \\
$-162.338 ;-57.071$
\end{tabular} & $\begin{array}{l}-102.273 \\
-182.895 ;-56.164\end{array}$ \\
\hline \multirow[t]{3}{*}{$Y \operatorname{Max}[\mathrm{cm}]$} & $6-9$ & \begin{tabular}{|l|}
1.430 \\
$0.780 ; 2.910$
\end{tabular} & \begin{tabular}{|l|}
1.185 \\
$0.780 ; 2.420$
\end{tabular} & - & $\begin{array}{l}1.550 \\
0.880 ; 2.520\end{array}$ \\
\hline & $10-13$ & \begin{tabular}{|l|}
0.940 \\
$0.470 ; 1.980$ \\
\end{tabular} & \begin{tabular}{|l|}
0.875 \\
$0.500 ; 1.600$ \\
\end{tabular} & - & \begin{tabular}{|l|}
1.280 \\
$0.750 ; 2.290$ \\
\end{tabular} \\
\hline & $14-18$ & $\begin{array}{l}0.950 \\
0.630 ; 1.800\end{array}$ & $\begin{array}{l}0.850 \\
0.460 ; 1.530\end{array}$ & - & $\begin{array}{l}1.160 \\
0.630 ; 2.250\end{array}$ \\
\hline \multirow[t]{3}{*}{$Y \operatorname{Min}[\mathrm{cm}]$} & $6-9$ & $\begin{array}{l}-1.530 \\
-2.980 ;-0.810\end{array}$ & $\begin{array}{l}-1.250 \\
-2.340 ;-0.750\end{array}$ & - & $\begin{array}{l}-1.560 \\
-2.990 ;-0.930\end{array}$ \\
\hline & $10-13$ & $\begin{array}{l}-1.030 \\
-1.990 ;-0.560\end{array}$ & $\begin{array}{l}-1.060 \\
-1.510 ;-0.540\end{array}$ & - & \begin{tabular}{|l|}
-1.330 \\
$-2.700 ;-0.690$ \\
\end{tabular} \\
\hline & $14-18$ & $\begin{array}{l}-0.930 \\
-2.070 ;-0.680\end{array}$ & $\begin{array}{l}-0.795 \\
-1.590 ;-0.490\end{array}$ & - & $\begin{array}{l}-1.180 \\
-2.090 ;-0.710\end{array}$ \\
\hline Symmetry $Y$ & $6-18$ & - & - & \begin{tabular}{|l|}
-96.257 \\
$-154.023 ;-64.162$ \\
\end{tabular} & $\begin{array}{l}-99.359 \\
-159.259 ;-58.852 \\
\end{array}$ \\
\hline \multirow[t]{3}{*}{ Range $X[\mathrm{~cm}]$} & $6-9$ & \begin{tabular}{|l|}
2.020 \\
$1.020 ; 4.900$
\end{tabular} & $\begin{array}{l}1.725 \\
0.820 ; 3.200\end{array}$ & - & $\begin{array}{l}2.270 \\
1.320 ; 4.380\end{array}$ \\
\hline & $10-13$ & $\begin{array}{l}1.460 \\
0.750 ; 2.790\end{array}$ & $\begin{array}{l}1.320 \\
0.820 ; 2.400\end{array}$ & - & \begin{tabular}{|l|}
1.650 \\
$0.780 ; 3.240$
\end{tabular} \\
\hline & $14-18$ & $\begin{array}{l}1.460 \\
0.630 ; 2.120\end{array}$ & \begin{tabular}{|l|}
1.045 \\
$0.600 ; 2.250$ \\
\end{tabular} & - & $\begin{array}{l}1.520 \\
0.750 ; 2.890 \\
\end{array}$ \\
\hline \multirow[t]{3}{*}{ Range $Y[\mathrm{~cm}]$} & $6-9$ & \begin{tabular}{|l|}
2.970 \\
$1.750 ; 5.370$
\end{tabular} & $\begin{array}{l}2.555 \\
1.480 ; 4.730\end{array}$ & - & \begin{tabular}{|l|}
3.230 \\
$1.930 ; 5.490$
\end{tabular} \\
\hline & $10-13$ & \begin{tabular}{|l|}
1.910 \\
$1.040 ; 4.420$
\end{tabular} & \begin{tabular}{|l|}
1.935 \\
$1.180 ; 3.060$
\end{tabular} & - & \begin{tabular}{|l|}
2.610 \\
$1.590 ; 4.650$
\end{tabular} \\
\hline & $14-18$ & $\begin{array}{l}2.040 \\
1.400 ; 4.030\end{array}$ & $\begin{array}{l}1.610 \\
0.970 ; 2.960\end{array}$ & - & $\begin{array}{l}2.330 \\
1.410 ; 4.230\end{array}$ \\
\hline \multirow[t]{3}{*}{$\begin{array}{l}\text { Circular sway } \\
\text { area }\left[\mathrm{cm}^{2}\right]\end{array}$} & $6-9$ & $\begin{array}{l}4.410 \\
2.360 ; 17.960\end{array}$ & $\begin{array}{l}3.765 \\
1.740 ; 8.980\end{array}$ & - & $\begin{array}{l}6.190 \\
2.790 ; 12.660\end{array}$ \\
\hline & $10-13$ & $\begin{array}{l}2.730 \\
0.990 ; 6.130\end{array}$ & $\begin{array}{l}2.545 \\
1.380 ; 4.340\end{array}$ & - & $\begin{array}{l}3.770 \\
1.490 ; 9.460\end{array}$ \\
\hline & $14-18$ & $\begin{array}{l}2.730 \\
0.990 ; 6.130\end{array}$ & $\begin{array}{l}1.920 \\
0.680 ; 4.740\end{array}$ & - & \begin{tabular}{|l|}
3.240 \\
$1.160 ; 8.340$ \\
\end{tabular} \\
\hline \multirow[t]{3}{*}{ PathLength [cm] } & $6-9$ & - & - & $\begin{array}{l}208.780 \\
135.150 ; 314.800\end{array}$ & $\begin{array}{l}213.930 \\
145.150 ; 309.900\end{array}$ \\
\hline & $10-13$ & - & - & $\begin{array}{l}129.540 \\
96.750 ; 174.040\end{array}$ & \begin{tabular}{|l|}
134.500 \\
$98.050 ; 172.550$
\end{tabular} \\
\hline & $14-18$ & - & - & $\begin{array}{l}89.590 \\
69.690 ; 122.640\end{array}$ & $\begin{array}{l}94.390 \\
73.110 ; 134.190\end{array}$ \\
\hline \multirow[t]{3}{*}{$V \operatorname{Avg}[\mathrm{cm} / \mathrm{s}]$} & $6-9$ & - & - & $\begin{array}{l}6.950 \\
4.510 ; 10.310 \\
\end{array}$ & $\begin{array}{l}7.130 \\
4.840 ; 10.330 \\
\end{array}$ \\
\hline & $10-13$ & - & - & \begin{tabular}{|l|}
4.300 \\
$3.220 ; 5.660$ \\
\end{tabular} & \begin{tabular}{|l|}
4.490 \\
$3.270 ; 5.750$ \\
\end{tabular} \\
\hline & $14-18$ & - & - & \begin{tabular}{|l|}
2.990 \\
$2.320 ; 4.090$
\end{tabular} & \begin{tabular}{|l|}
3.150 \\
$2.440 ; 4.470$
\end{tabular} \\
\hline \multirow[t]{3}{*}{ Area95 $\left[\mathrm{cm}^{2}\right]$} & $6-9$ & \begin{tabular}{|l|}
3.160 \\
$1.220 ; 21.870$
\end{tabular} & $\begin{array}{l}2.540 \\
0.900 ; 6.940\end{array}$ & - & \begin{tabular}{|l|}
3.860 \\
$1.660 ; 14.330$
\end{tabular} \\
\hline & $10-13$ & $\begin{array}{l}2.180 \\
0.570 ; 5.720\end{array}$ & \begin{tabular}{|l|}
1.690 \\
$0.500 ; 4.060$
\end{tabular} & - & $\begin{array}{l}2.820 \\
1.030 ; 10.190\end{array}$ \\
\hline & $14-18$ & $\begin{array}{l}1.750 \\
0.800 ; 5.520\end{array}$ & $\begin{array}{l}1.230 \\
0.390 ; 3.140\end{array}$ & - & $\begin{array}{l}2.290 \\
0.660 ; 5.820\end{array}$ \\
\hline \multirow[t]{3}{*}{ WCOP } & $6-9$ & - & - & \multirow[t]{3}{*}{$\begin{array}{l}17.391 \\
-9.091 ; 46.383\end{array}$} & \begin{tabular}{|l|}
15.629 \\
$-13.646 ; 51.099$
\end{tabular} \\
\hline & $10-13$ & - & - & & \begin{tabular}{|l|}
23.459 \\
$-7.609 ; 48.045$
\end{tabular} \\
\hline & $14-18$ & - & - & & \begin{tabular}{|l|}
25.595 \\
$-15.108 ; 49.153$
\end{tabular} \\
\hline
\end{tabular}

In the cases of significant between-gender differences, the variables are presented separately for boys and girls. If the differences between genders were non-significant, the data were pooled together and medians and percentiles were calculated for the entire group. 
Table 6. Median and 5\% and 95\% percentiles of the balance variables in group of 228 healthy subjects (111 boys and 117 girls). Test: AP test

\begin{tabular}{|c|c|c|c|c|c|}
\hline \multirow{2}{*}{ Variable } & Age [years] & Boys & Girls & Together & \multirow{2}{*}{$\begin{array}{c}\text { Together } \\
\text { Eyes closed }\end{array}$} \\
\hline & \multicolumn{4}{|c|}{ Eyes open } & \\
\hline 1 & 2 & 3 & 4 & 5 & 6 \\
\hline \multirow[t]{3}{*}{$X \operatorname{Max}[\mathrm{cm}]$} & $6-9$ & $\begin{array}{l}2.265 \\
1.400 ; 4.200\end{array}$ & $\begin{array}{l}2.555 \\
1.860 ; 5.170\end{array}$ & - & $\begin{array}{l}3.700 \\
1.930 ; 5.250\end{array}$ \\
\hline & $10-13$ & $\begin{array}{l}2.440 \\
1.540 ; 4.150\end{array}$ & $\begin{array}{l}2.175 \\
1.250 ; 3.510 \\
\end{array}$ & - & $\begin{array}{l}2.620 \\
1.510 ; 4.470 \\
\end{array}$ \\
\hline & $14-18$ & \begin{tabular}{|l|}
2.140 \\
$1.370 ; 3.710$
\end{tabular} & \begin{tabular}{|l|}
1.890 \\
$1.170 ; 3.280$
\end{tabular} & - & \begin{tabular}{|l|}
2.310 \\
$1.480 ; 4.570$
\end{tabular} \\
\hline \multirow[t]{3}{*}{$X \operatorname{Min}[\mathrm{cm}]$} & $6-9$ & - & - & $\begin{array}{l}-2.610 \\
-3.760 ;-1.450\end{array}$ & $\begin{array}{l}-3.470 \\
-6.090 ;-2.330\end{array}$ \\
\hline & $10-13$ & - & - & $\begin{array}{l}-2.210 \\
-4.270 ;-1.280 \\
\end{array}$ & $\begin{array}{l}-2.410 \\
-3.900 ;-1.520 \\
\end{array}$ \\
\hline & $14-18$ & - & - & $\begin{array}{l}-1.985 \\
-3.280 ;-1.110 \\
\end{array}$ & $\begin{array}{l}-2.300 \\
-4.180 ;-1.490 \\
\end{array}$ \\
\hline Symmetry $X$ & $6-18$ & - & - & $\begin{array}{l}-100.000 \\
-160.000 ;-65.517\end{array}$ & $\begin{array}{l}-100.000 \\
-158.511 ;-69.536\end{array}$ \\
\hline \multirow[t]{3}{*}{$Y \operatorname{Max}[\mathrm{cm}]$} & $6-9$ & - & - & $\begin{array}{l}7.930 \\
5.860 ; 10.040\end{array}$ & $\begin{array}{l}7.730 \\
6.270 ; 10.350\end{array}$ \\
\hline & $10-13$ & - & - & $\begin{array}{l}8.790 \\
6.350 ; 10.820 \\
\end{array}$ & $\begin{array}{l}8.800 \\
6.900 ; 10.620\end{array}$ \\
\hline & $14-18$ & - & - & $\begin{array}{l}9.280 \\
7.490 ; 10.960 \\
\end{array}$ & $\begin{array}{l}9.150 \\
7.480 ; 11.020\end{array}$ \\
\hline \multirow[t]{3}{*}{$Y \operatorname{Min}[\mathrm{cm}]$} & $6-9$ & - & - & $\begin{array}{l}-6.800 \\
-8.770 ;-5.140\end{array}$ & $\begin{array}{l}-7.160 \\
-8.260 ;-5.240\end{array}$ \\
\hline & $10-13$ & - & - & $\begin{array}{l}-8.330 \\
-10.020 ;-6.030 \\
\end{array}$ & \begin{tabular}{|l|}
-8.330 \\
$-10.310 ;-6.080$ \\
\end{tabular} \\
\hline & $14-18$ & - & - & $\begin{array}{l}-8.890 \\
-10.370 ;-6.720 \\
\end{array}$ & \begin{tabular}{|l|}
-8.690 \\
$-11.230 ;-6.610$ \\
\end{tabular} \\
\hline \multirow[t]{3}{*}{ Symmetry $Y$} & $6-9$ & - & - & $\begin{array}{l}-115.340 \\
-148.208 ;-95.028\end{array}$ & \multirow[t]{3}{*}{$\begin{array}{l}-107.299 \\
-129.370 ;-87.389\end{array}$} \\
\hline & $10-13$ & - & - & $\begin{array}{l}-106.740 \\
-126.177 ;-90.525 \\
\end{array}$ & \\
\hline & $14-18$ & - & - & $\begin{array}{l}-105.830 \\
-128.808 ;-83.475 \\
\end{array}$ & \\
\hline \multirow[t]{3}{*}{ Range $X[\mathrm{~cm}]$} & $6-9$ & - & - & \begin{tabular}{|l|}
5.220 \\
$3.050 ; 7.810$ \\
\end{tabular} & \begin{tabular}{|l|}
6.950 \\
$4.330 ; 10.400$
\end{tabular} \\
\hline & $10-13$ & - & - & $\begin{array}{l}4.640 \\
2.840 ; 7.690 \\
\end{array}$ & $\begin{array}{l}5.120 \\
2.970 ; 8.620\end{array}$ \\
\hline & $14-18$ & - & - & $\begin{array}{l}3.990 \\
2.590 ; 6.820\end{array}$ & $\begin{array}{l}4.680 \\
3.140 ; 8.190\end{array}$ \\
\hline \multirow[t]{3}{*}{ Range $Y[\mathrm{~cm}]$} & $6-9$ & - & - & $\begin{array}{l}14.550 \\
11.510 ; 18.330 \\
\end{array}$ & $\begin{array}{l}14.520 \\
12.100 ; 18.220\end{array}$ \\
\hline & $10-13$ & & & $\begin{array}{l}17.420 \\
12.890 ; 20.360 \\
\end{array}$ & $\begin{array}{l}17.070 \\
12.980 ; 19.810 \\
\end{array}$ \\
\hline & $14-18$ & & & $\begin{array}{l}18.220 \\
14.360 ; 20.920\end{array}$ & $\begin{array}{l}17.930 \\
14.230 ; 21.500\end{array}$ \\
\hline \multirow[t]{3}{*}{$\begin{array}{l}\text { Circular sway } \\
\text { area }\left[\mathrm{cm}^{2}\right]\end{array}$} & $6-9$ & - & - & $\begin{array}{l}54.790 \\
28.320 ; 83.470\end{array}$ & \multirow[t]{3}{*}{$\begin{array}{l}58.620 \\
33.370 ; 109.140\end{array}$} \\
\hline & $10-13$ & & & $\begin{array}{l}54.280 \\
25.170 ; 92.780\end{array}$ & \\
\hline & $14-18$ & & & $\begin{array}{l}48.265 \\
25.320 ; 76.390 \\
\end{array}$ & \\
\hline PathLength [cm] & $6-18$ & $\begin{array}{l}376.200 \\
283.320 ; 492.230\end{array}$ & $\begin{array}{l}398.150 \\
273.690 ; 532.390\end{array}$ & - & $\begin{array}{l}391.490 \\
273.580 ; 526.070\end{array}$ \\
\hline
\end{tabular}




\begin{tabular}{|c|c|c|c|c|c|}
\hline 1 & 2 & 3 & 4 & 5 & 6 \\
\hline$V \operatorname{Avg}[\mathrm{cm} / \mathrm{s}]$ & $6-18$ & - & - & $\begin{array}{l}12.865 \\
9.200 ; 17.420\end{array}$ & $\begin{array}{l}13.050 \\
9.320 ; 17.540\end{array}$ \\
\hline Area95 $\left[\mathrm{cm}^{2}\right]$ & $6-18$ & - & - & \begin{tabular}{|l|}
80.255 \\
$38.460 ; 130.280$ \\
\end{tabular} & $\begin{array}{l}90.210 \\
46.800 ; 173.400 \\
\end{array}$ \\
\hline \multirow[t]{3}{*}{ WCOP } & $6-9$ & - & - & $\begin{array}{l}47.193 \\
26.476 ; 61.431\end{array}$ & $\begin{array}{l}38.824 \\
12.793 ; 53.025\end{array}$ \\
\hline & $10-13$ & - & - & $\begin{array}{l}58.660 \\
41.188 ; 68.041\end{array}$ & $\begin{array}{l}53.982 \\
35.958 ; 69.729\end{array}$ \\
\hline & $14-18$ & - & - & $\begin{array}{l}63.104 \\
47.450 ; 73.281\end{array}$ & $\begin{array}{l}58.748 \\
42.401 ; 68.608\end{array}$ \\
\hline
\end{tabular}

In the cases of significant between-gender differences, the variables are presented separately for boys and girls. If the differences between genders were non-significant, the data were pooled together and medians and percentiles were calculated for the entire group.

Table 7. Median and 5\% and $95 \%$ percentiles of the balance variables in group of 228 healthy subjects (111 boys and 117 girls). Test: ML test

\begin{tabular}{|c|c|c|c|}
\hline \multirow{2}{*}{ Variable } & \multirow{2}{*}{ Age } & Together & Together \\
\hline & & Eyes open & Eyes closed \\
\hline 1 & 2 & 3 & 4 \\
\hline \multirow[t]{3}{*}{$X \operatorname{Max}[\mathrm{cm}]$} & $6-9$ & $\begin{array}{l}11.500 \\
7.290 ; 12.780 \\
\end{array}$ & $\begin{array}{l}10.660 \\
8.200 ; 12.250 \\
\end{array}$ \\
\hline & $10-13$ & $\begin{array}{l}10.750 \\
8.560 ; 13.290\end{array}$ & $\begin{array}{l}10.630 \\
8.900 ; 13.530\end{array}$ \\
\hline & $14-18$ & $\begin{array}{l}11.540 \\
8.980 ; 13.890\end{array}$ & $\begin{array}{l}11.415 \\
8.980 ; 14.340\end{array}$ \\
\hline \multirow[t]{3}{*}{$X \operatorname{Min}[\mathrm{cm}]$} & $6-9$ & $\begin{array}{l}-10.080 \\
-13.130 ;-7.150\end{array}$ & $\begin{array}{l}-9.820 \\
-12.560 ;-7.970\end{array}$ \\
\hline & $10-13$ & $\begin{array}{l}-10.490 \\
-13.670 ;-8.220 \\
\end{array}$ & $\begin{array}{l}-10.880 \\
-13.900 ;-8.690 \\
\end{array}$ \\
\hline & $14-18$ & \begin{tabular}{|l|}
-11.630 \\
$-13.920 ;-8.430$ \\
\end{tabular} & $\begin{array}{l}-11.265 \\
-13.590 ;-8.550 \\
\end{array}$ \\
\hline Symmetry $X$ & $6-18$ & $\begin{array}{l}-100.816 \\
-118.279 ;-86.024\end{array}$ & $\begin{array}{l}-100.295 \\
-118.066 ;-86.842\end{array}$ \\
\hline \multirow[t]{3}{*}{$Y \operatorname{Max}[\mathrm{cm}]$} & $6-9$ & $\begin{array}{l}.110 \\
1.970 ; 4.200\end{array}$ & \multirow[t]{3}{*}{$\begin{array}{l}3.435 \\
2.200 ; 5.590\end{array}$} \\
\hline & $10-13$ & $\begin{array}{l}.140 \\
1.790 ; 4.790\end{array}$ & \\
\hline & $14-18$ & $\begin{array}{l}2.780 \\
1.840 ; 4.150\end{array}$ & \\
\hline$Y \operatorname{Min}[\mathrm{cm}]$ & & $\begin{array}{l}-2.760 \\
-4.060 ;-1.860\end{array}$ & $\begin{array}{l}-3.285 \\
-4.900 ;-2.000\end{array}$ \\
\hline \multirow[t]{3}{*}{ Symmetry $Y$} & $6-9$ & $\begin{array}{l}-106.020 \\
-161.753 ;-81.452 \\
\end{array}$ & $\begin{array}{l}-111.803 \\
-168.269 ;-74.005\end{array}$ \\
\hline & $10-13$ & $\begin{array}{l}-108.519 \\
-160.400 ;-77.778\end{array}$ & $\begin{array}{l}-104.208 \\
-152.263 ;-58.663\end{array}$ \\
\hline & $14-18$ & $\begin{array}{l}-102.410 \\
-154.941 ;-66.319\end{array}$ & $\begin{array}{l}-103.515 \\
-145.631 ;-70.488\end{array}$ \\
\hline \multirow[t]{3}{*}{ Range $X[\mathrm{~cm}]$} & $6-9$ & $\begin{array}{l}20.230 \\
14.520 ; 25.460\end{array}$ & $\begin{array}{l}20.310 \\
16.380 ; 24.330\end{array}$ \\
\hline & $10-13$ & $\begin{array}{l}21.170 \\
17.090 ; 26.780\end{array}$ & $\begin{array}{l}21.370 \\
18.100 ; 27.430\end{array}$ \\
\hline & $14-18$ & $\begin{array}{l}23.280 \\
17.290 ; 27.540\end{array}$ & $\begin{array}{l}22.480 \\
17.370 ; 27.410\end{array}$ \\
\hline Range $Y[\mathrm{~cm}]$ & & \begin{tabular}{|l}
5.820 \\
$3.850 ; 8.240$
\end{tabular} & $\begin{array}{l}6.735 \\
4.230 ; 9.790\end{array}$ \\
\hline
\end{tabular}




\begin{tabular}{|c|c|c|c|}
\hline 1 & 2 & 3 & 4 \\
\hline \multirow[t]{3}{*}{$\begin{array}{l}\text { Circular sway } \\
\text { area }\left[\mathrm{cm}^{2}\right]\end{array}$} & $6-9$ & $\begin{array}{l}102.080 \\
56.810 ; 147.350\end{array}$ & \multirow[t]{3}{*}{$\begin{array}{l}124.100 \\
79.730 ; 190.830\end{array}$} \\
\hline & $10-13$ & $\begin{array}{l}107.940 \\
62.810 ; 172.430\end{array}$ & \\
\hline & $14-18$ & $\begin{array}{l}105.620 \\
73.090 ; 165.580\end{array}$ & \\
\hline PathLength [cm] & & $\begin{array}{l}505.420 \\
357.260 ; 722.410\end{array}$ & $\begin{array}{l}504.140 \\
349.050 ; 686.650\end{array}$ \\
\hline V Avg $[\mathrm{cm} / \mathrm{s}]$ & & $\begin{array}{l}16.650 \\
11.290 ; 22.840\end{array}$ & $\begin{array}{l}16.800 \\
11.640 ; 22.890\end{array}$ \\
\hline \multirow[t]{3}{*}{ Area95 $\left[\mathrm{cm}^{2}\right]$} & $6-9$ & $\begin{array}{l}129.340 \\
66.500 ; 208.520\end{array}$ & $\begin{array}{l}155.720 \\
102.090 ; 274.220\end{array}$ \\
\hline & $10-13$ & $\begin{array}{l}148.770 \\
68.200 ; 246.840\end{array}$ & $\begin{array}{l}176.890 \\
77.460 ; 319.260\end{array}$ \\
\hline & $14-18$ & $\begin{array}{l}154.550 \\
89.690 ; 257.590\end{array}$ & $\begin{array}{l}171.360 \\
106.650 ; 271.660\end{array}$ \\
\hline \multirow[t]{3}{*}{ WCOP } & $6-9$ & $\begin{array}{l}54.822 \\
39.391 ; 64.986\end{array}$ & \multirow[t]{3}{*}{$\begin{array}{l}51.821 \\
36.119 ; 63.859\end{array}$} \\
\hline & $10-13$ & $\begin{array}{l}56.333 \\
43.041 ; 65.133\end{array}$ & \\
\hline & $14-18$ & $\begin{array}{l}59.798 \\
47.282 ; 69.937\end{array}$ & \\
\hline
\end{tabular}

In the cases of significant between-gender differences, the variables are presented separately for boys and girls. If the differences between genders were nonsignificant, the data were pooled together and medians and percentiles were calculated for the entire group.

\section{Discussion}

The primary aim of this study was to verify the existence of between-gender differences in balance measures in the group of 6- to18-year-old subjects. During quiet standing with eyes open, the median values of evaluated balance parameters indicated better balance control in girls than in boys. The results showed lower values of voluntary sway to the left and right, forward and backward, as well as sway ranges in AP and ML planes in girls than in boys of all age groups. One of the most interesting findings was a lack of statistically significant differences between genders in test with eyes closed. This finding suggests that vision, an important sensory input especially in younger children, is differently used by females and males in balance tasks. During quiet standing with eyes open (in this test most variables were gender dependent), the median values of evaluated balance parameters indicated better balance control in girls. The results (Table 5) show lower values of voluntary sway to the left and right, forward and backward, as well as sway ranges in AP and ML planes in girls than in boys of all age groups. This result is similar to the results of the study of Pannicia et al. [12], who also found out that females 9 to 12 years old had better postural stability when visual information is present, i.e., with eyes open.

The results of the study confirm the maturation of the balance which occurs with age. The balance parameters during quiet standing in both conditions (eyes open and eyes closed) decrease with age, showing better control over body posture. The correlation coefficients of balance measures with age are mostly weak or medium, but in the case of two parameters (path length and mean velocity), the correlations are strong (respectively: $r=-0.875$ and $r=-0.883$ in eyes open condition, and $r=-0.813$ and $r=-0.798$ in eyes closed condition). Path length decreases from $208.78 \mathrm{~cm}$ in the case of children aged 6 to 9 years old, to $89.59 \mathrm{~cm}$ in the case of children aged 14 to 18 years old in eyes open condition, in the case of eyes closed condition the decrease was from $213.93 \mathrm{~cm}$ to $94.39 \mathrm{~cm}$, thus in both conditions the decrease was of nearly $60 \%$. In the case of mean velocity the decrease was of similar amount: from $6.95 \mathrm{~cm} / \mathrm{s}$ in children of 6 to 9 years old to $2.99 \mathrm{~cm} / \mathrm{s}$ in children of 14 to 18 years old in eyes open condition, and from $7.13 \mathrm{~cm} / \mathrm{s}$ in children of 6 to 9 years old 
to $3.15 \mathrm{~cm} / \mathrm{s}$ in children 14 to 19 years old in eyes closed condition.

The present study shows that parameters of AP and ML tests increased with age, which suggests better control over the body, as the subjects can safely lean out more in sagittal or frontal plane when they are older. Partially this increase can be also connected with increase of body height and gradual shift up of the body centre of mass, which happens with age.

Children show different postural control strategy than young adults. Children relay more on visual feedback and control, while young adults relay primarily on somatosensory inputs. Of the three sensory inputs in children, the vestibular system seems to be less effective in postural control [4].

The results of this study are in agreement with other studies which assessed changes of balance parameters in children and adolescents with age. Libardoni et al. [8] found that age is an important variable responsible for the development of postural control. In their study, younger children aged 8 to 10 had lower balance scores than older children of 11 and 12 years old. Similar results were found also by Pannicia et al. [12], who found out that balance stability scores improved between 2 and 13 years of age.

In AP test (antero-posterior maximal voluntary sways) of the present study, maximal voluntary mediolateral displacement of COP was higher in girls than in boys only in the youngest group, aged 6 to 9. In two older groups, median value of this parameter was higher in boys. However, in 10- to 13-year-olds, there is no essential body height difference between boys and girls [6]. The path length in this test (with eyes open) was not age dependent, but was gender dependent, with median values higher in females than in males. This suggests that females performed more corrective movements during voluntary sways in frontal plane than the males. In ML test, there was differences between girls and boys, but some parameters were age dependent: the range of the sways in the frontal plane increased with age, as well as circular sway area and Area95.

\section{Conclusion}

Quiet standing postural sway characteristics depended on gender under normal visual conditions and it was similar in boys and girls under visual deprivation conditions. The vision was differently used by females and males in balance tasks. Static postural stability improved with age, regardless of visual conditions. The results of this study can be used in clini- cal everyday practice to assess possible balance problems in children and adolescents, and to evaluate the effects of the treatment and its influence on the balance abilities of the patients.

\section{Acknowledgements}

The study was supported by research grant Virtual Balance Clinic VB-Clinic founded by NCBR Poland (STRATEGMED3/306011/1/ NCBR/2017) and The Children's Memorial Health Institute's statutory research task No. W/17.

\section{Ethical approval}

The protocol and the study was approved by Bioethical Committee of The Children's Memorial Health Institute, Warsaw, Poland, agreement 183/KBE/2015 and Bioethical Committee of Medical University of Warsaw, agreement $\mathrm{KB} / 28 / 2014$.

\section{References}

[1] Assaiante C., Development of locomotor balance control in healthy children, Neurosci. Biobehav. Rev., 1998, 22, 527-532.

[2] Bohannon R., LEARY K., Standing balance and function over the course of acute rehabilitation, Arch. Phys. Med. Rehabil., 1995, 76, 994-996

[3] EMERY C., Is there a clinical standing balance measurement appropriate for use in sports medicine? A review of literature, J. Sci. and Med. in Sport, 2003, 6, 492-504.

[4] Ferber-Viart C., Ionescu E., Morlet T., Froehlich P., DuBREUIL C., Balance in healthy individuals assessed with Equitest: maturation and normative data for children and young adults, Int. J. Pediatr. Otorhinolaryngology, 2007, 71, 1041-1046.

[5] Gera G., Freeman D., Blackinton M., Horak F., King L. et al., Identification of balance deficits in people with Parkinson disease; is the sensory organization enough?, Int. J. Phys. Med. \& Rehabil., 2016, 4, pii:322.

[6] KuŁaga Z., RÓŻDŻYŃSKA-ŚWiĄTKOWSKA A., GRAJDA A., Gurzkowska B., WoJTyŁo M., Góźdź M., ŚwiądeK-LeŚNIAK A., Litwin M., Percentile charts for growth and nutritional status assessment in Polish children and adolescents from birth to 18 year of age, Standardy Medyczne Pediatria, 2015, 12, 119-135.

[7] LEBIEDOWSKA M., SYCZEWSKA M., Invariant sway properties in children, Gait and Posture, 2000, 12, 200-204.

[8] Libardoni T., da Silveira C., Singhorim L., De Oliveira A., Dos SAntos M., SANTOS G., Reference values and equations reference of balance for children of 8 to 12 years, Gait and Posture, 2018, 60, 122-127.

[9] LUDWIG O., Interrelationship between postural balance and body posture in children and adolescents, J. Phys. Ther. Sci., 2017, 29, 1154-1158.

[10] Mraz M., Nowacka U., Skrzek A., Mraz M., DęBIEC-BąK A., Sidorowska M., Postural stability of women at the age of 8-22 on the basis of posturographic examinations, Fizjoterapia, 2010, 18, 35-43. 
[11] Opala-Berdzik A., GŁowacka M., Wilusz K., Kolacz P., SZYDŁo K., JURAS G., Quiet standing postural sway of 10- to 13-year-old, national level, female acrobatic gymnasts, Acta Biomech. Bioeng., 2018, 20, 117-123.

[12] Pannicia M., Wilson K., Hunt A., Keightley M., ZabJeK K., TAha T., GaGnOn I., ReED N., Postural stability in healthy child and youth athletes: The effect of age, sex, and concussion-related factors on performance, Sport Health, 2018, 10, 175-182.

[13] Ringhof S., SteIN T., Biomechanical assessment of dynamic balance: Specificity of different balance tests, Hum. Mov. Sci., 2018, 58, 140-147.
[14] Schober P., Boer C., Schwarte L.A., Correlation Coefficients: Appropriate Use and Interpretation, Anesth. Analg. 2018, 126, 1763-1768.

[15] Sobera M., Siedlecka B., Syczewska M., Posture control development in children aged 2-7 years old, based on the changes of repeatability of the stability indices, Neurosci. Lett., 2011, 491, 13-17.

[16] Syczewska M., Dembowska-Bagińska B., Perek-Polnik M., Kalinowska M., PereK D., Postural sway in children and young adults, survivors of CNS tumours, Adv. Med. Sci., 2008, 53, 256-262. 